\title{
No low-frequency turn-over in the spectra of millisecond pulsars
}

\author{
A. D. Kuzmin and B. Ya. Losovsky
}

\author{
Pushchino Radio Astronomy Observatory, Astro Space Center, Lebedev Physical Institute, Pushchino, \\ Moscow Region, 142290, Russia
}

Received 28 July 2000 / Accepted 22 December 2000

\begin{abstract}
We present the results of the first low frequency measurements of the flux densities of a large sample of millisecond pulsars (MSPs) at 102 and $111 \mathrm{MHz}$. Combining our observations with data at higher frequencies borrowed from the literature, we constructed the spectra of $30 \mathrm{MSPs}$ in the 0.1 to $4.8 \mathrm{GHz}$ frequency range. Our flux density measurements extend the known spectra of MSPs to the lowest frequency to date. The coverage of a low frequency range allows us to search for low frequency turn-over, which is often observed for normal pulsars. We find that spectra of MSPs differ from those of normal pulsars, showing no low-frequency turn-over typical of normal pulsars. We suggest that the geometry of the radio emission region of MSPs differs from that of normal pulsars: the magnetic field configuration in MSPs' magnetospheres may deviate from that of a pure dipole and/or the radio emission region may be radially compressed. Monochromatic luminosities at this low frequency and the integral luminosity over the $0.1-4.8 \mathrm{GHz}$ frequency range were determined and their dependence on other pulsar parameters analyzed.
\end{abstract}

Key words. stars: pulsars: general - stars: magnetic fields stars: neutrons - radio continuum: stars

\section{Introduction}

Spectra are one of the main pulsar characteristics and are of great importance to understand the mechanism of pulsar radio emission.

Millisecond pulsars (MSPs) are believed to be a special population of pulsars, which are distinguished from "normal" ones by period, period derivative, magnetic field strength, age and evolutionary history. One may expect that the radio emission characteristics of MSPs will be different from those of normal pulsars as well. However, comparative studies of the radio emission characteristics between MSPs and normal pulsars have so far shown only slight differences.

Foster et al. (1991) have obtained the spectra of four MSPs in the frequency range between 425 and $3000 \mathrm{MHz}$. They yield spectral indices between -1.3 to -2.6 . They noted that the mean spectral index of these MSPs is steeper than the average spectral index of slow period pulsars. Lorimer et al. (1995) obtained spectra for 19 MSPs and also concluded that they are slightly steeper than normal pulsars.

Kramer et al. (1998) and Xilouris et al. (1998) presented a collection of flux density measurements of 27 MSPs at $1.4 \mathrm{GHz}$. Comparing to data borrowed from literature they concluded that the spectra of normal

Send offprint requests to: A. Kuzmin,

e-mail: akuzmin@prao.psn.ru and MSPs show great similarities in the frequency range between 0.4 and $1.4 \mathrm{GHz}$. A similar conclusion was reached by Toscano et al. (1998) for a large sample of MSPs in the southern hemisphere at frequencies between 400 and 1600 MHz. Kijak et al. (1997) and Kramer et al. (1999) extended the known spectra of MSPs to the highest frequency, $4.8 \mathrm{GHz}$. The coverage of this high-frequency band allows them to search for frequency breaks, often observed for normal pulsars around $1 \mathrm{GHz}$. Their results suggest that, unlike the normal pulsars, MSPs spectra from 0.4 to $4.8 \mathrm{GHz}$ can be largely described by a single power law, $F(\nu) \propto \nu^{\alpha}$. They report also that there is no significant difference in the mean spectral index $\alpha$ of MSPs and normal pulsars. Kramer et al. (1999) conclude also that MSPs tend to be slightly less luminous than normal pulsars.

These studies concentrate mainly on high radio frequencies, while there is a lack of data at lower radio frequencies. Especially interesting is the low-frequency part of pulsar spectra, which typically shows a low frequency turn-over (Sieber 1973; Bruk et al. 1978; Kuzmin et al. 1978, Izvekova et al. 1981). Malofeev (2000) reported that 102 slow pulsars among 151 with studied spectral behaviour show a low-frequency spectral turn-over.

The frequency of the maximum of the spectra of normal pulsars, $\nu_{\max }$, depends on pulsar period as $\nu_{\max }=$ $120 P^{-0.36}$, where $\nu$ is in $\mathrm{MHz}$ and $P$ in sec (Malofeev \& Malov 1980; Malofeev 1996). Therefore, one may expect 
that for MSPs, a low-frequency turn-over should be obvious: for periods $P \leq 50 \mathrm{~ms}$ the frequency of the maximum of spectra is expected to be $\nu_{\max } \geq 350 \mathrm{MHz}$. Consequently, at our frequency $\approx 100 \mathrm{MHz}$ all MSPs should be in the low-frequency turn-over region.

Erickson \& Mahoney (1985) obtained the spectrum of PSR B1937+21 in continuum radio emission from $10 \mathrm{MHz}$ to $3 \mathrm{GHz}$ that showed no low-frequency turn-over.

Kuzmin et al. (1990) and Kuzmin \& Losovsky (1996) made the first observations of pulsed emission of MSPs PSR B1855+09 and PSR J2145-0750 at the low frequency $102 \mathrm{MHz}$ and reported no evidence of a low frequency turn-over.

Kuzmin \& Losovsky (2000a, 2000b) undertook flux density measurements of $18 \mathrm{MSPs}$ at $102 \mathrm{MHz}$, which extends our knowledge of MSPs spectra to the lowest frequency where MSP observations have been performed thus far. They claimed that spectra of MSPs have no low frequency turn-over.

We have now extended the low frequency observations to include $30 \mathrm{MSPs}$ and combined our observations with higher frequency data to study their spectra and luminosity. We compare our results with a sample of normal pulsars, searching for similarities and differences in their spectra and luminosities.

\section{Observations}

The observations were performed between 1996 and 2000 with the Large Phased Array BSA radio telescope in Pushchino Radio Astronomy Observatory. Linear polarization was received. A filter bank of $32 \times 5 \mathrm{kHz}$ filters centered at 102 or $111 \mathrm{MHz}$ was used. The sampling interval was set between 0.128 and $0.64 \mathrm{~ms}$ depending on the pulsar period and the dispersion measure. All observations were time referenced to the Observatory rubidium master clock, which in turn was monitored against the National Time Standard via a TV timing signal. Each integrated profile was formed by the synchronous integration of individual pulses with the topocentric pulsar period. Each pulsar observational session was $4 / \cos \delta$ minutes, where $\delta$ is the declination of the pulsar. This interval is larger than the diffractive interstellar scintillation time scale of $\sim 1$ min (Malofeev 2000) Therefore, the effect of diffractive interstellar scintillation is smoothed during each observing session. The refractive scintillation time scale $t_{\mathrm{r}}$ was estimated by interpolation of Gupta et al. (1993) $74 \mathrm{MHz}$ data and Stinebring et al. (2000) $610 \mathrm{MHz}$ data. For our sources, $t_{\mathrm{r}}$ differs depending on the $D M$ of pulsars. For pulsars with small DM such as PSR J1744-1134, it is estimated to be about 2 days, while for large $D M$ pulsars such as PSR J0218+4232, B1620-26 and B2127+11C, it is estimated to be about 1 year. To reduce the influence of these scintillations, we averaged several observational sessions in our four year observational time span.

During off-line data reduction, the signal was cleaned of radio interference. Data from several sessions were
Table 1. Observational parameters

\begin{tabular}{|c|c|c|c|c|c|c|}
\hline$\overline{\mathrm{PSR}}$ & $\begin{array}{c}P \\
\mathrm{~ms}\end{array}$ & $\begin{array}{c}\dot{P} \\
10^{-20} \mathrm{~s} / \mathrm{s}\end{array}$ & $\begin{array}{c}D M \\
\text { pc } \mathrm{cm}^{-3}\end{array}$ & $\begin{array}{c}\nu \\
\mathrm{MHz}\end{array}$ & $\bar{M}$ & $\begin{array}{c}N \\
10^{3}\end{array}$ \\
\hline $\mathrm{J} 0030+0451$ & 4.86 & 1.0 & 4.3 & 111 & 35 & 622 \\
\hline J0034-0534 & 1.87 & 0.67 & 13.7 & 102 & 4 & 460 \\
\hline \multirow[t]{2}{*}{ J0218+4232 } & 2.33 & 7.5 & 61.2 & 102 & 7 & 883 \\
\hline & & & & 111 & 56 & 2952 \\
\hline J0613-0200 & 3.06 & 1.1 & 38.8 & 102 & 16 & 1130 \\
\hline J0621+1002 & 28.85 & 4 & 36.6 & 111 & 23 & 70 \\
\hline J0751+1807 & 3.47 & 0.8 & 30.2 & 102 & 8 & 520 \\
\hline $\mathrm{J} 1012+5307$ & 5.25 & 1.46 & 9.0 & 102 & 20 & 136 \\
\hline $\mathrm{J} 1022+1001$ & 16.45 & 4.2 & 10.2 & 102 & 17 & 226 \\
\hline J1024-0719 & 5.16 & 1.84 & 6.4 & 102 & 8 & 337 \\
\hline $\mathrm{B} 1257+12$ & 6.21 & 11.4 & 10.1 & 102 & 21 & 745 \\
\hline $\mathrm{B} 1310+18$ & 33.16 & - & 24.0 & 102 & 30 & 202 \\
\hline $\mathrm{J} 1518+4904$ & 40.93 & 3 & 11.6 & 102 & 20 & 161 \\
\hline B1534+12 & 37.90 & 243 & 11.6 & 102 & 22 & 128 \\
\hline \multirow[t]{2}{*}{ B1620-26 } & 11.07 & 79.04 & 62.8 & 102 & 15 & 327 \\
\hline & & & & 111 & 42 & 474 \\
\hline $\mathrm{B} 1639+36 \mathrm{~A}$ & 10.37 & - & 30.3 & 102 & 41 & 1054 \\
\hline $\mathrm{J} 1640+2224$ & 3.16 & 0.29 & 18.4 & 111 & 35 & 1057 \\
\hline $\mathrm{J} 1713+0747$ & 4.57 & 0.85 & 15.9 & 102 & 20 & 948 \\
\hline J1730-2304 & 8.12 & 1.9 & 9.6 & 102 & 44 & 1267 \\
\hline J1744-1134 & 4.09 & 0.86 & 3.1 & 111 & 3 & 508 \\
\hline $\mathrm{B} 1855+09$ & 5.36 & 1.78 & 13.3 & 102 & 20 & 814 \\
\hline J1911-1114 & 3.62 & 1.34 & 30.9 & 102 & 23 & 1396 \\
\hline $\mathrm{J} 2019+2425$ & 3.93 & 0.70 & 17.2 & 102 & 17 & 1020 \\
\hline $\mathrm{J} 2033+1736$ & 5.94 & - & 25.2 & 102 & 13 & 494 \\
\hline J2051-0827 & 4.51 & 1.3 & 20.7 & 111 & 22 & 402 \\
\hline $\mathrm{B} 2127+11 \mathrm{C}$ & 30.52 & 499 & 67.1 & 111 & 7 & 19 \\
\hline J2145-0750 & 16.05 & 3 & 9.0 & 102 & 21 & 283 \\
\hline $\mathrm{J} 2229+2643$ & 2.97 & 0.19 & 23.0 & 102 & 24 & 1903 \\
\hline \multirow[t]{2}{*}{$\mathrm{J} 2235+1506$} & 59.76 & 15.76 & 18.1 & 102 & 7 & 26 \\
\hline & & & & 111 & 17 & 44 \\
\hline $\mathrm{J} 2317+1439$ & 3.44 & 0.24 & 21.9 & 102 & 32 & 2074 \\
\hline $\mathrm{J} 2322+2057$ & 4.80 & 0.97 & 13.4 & 102 & 17 & 818 \\
\hline
\end{tabular}

combined to increase the signal-to-noise ratio and to reduce the influence of polarization and scintillation.

Table 1 lists the pulsars with their periods $P$, period derivatives $\dot{P}$, dispersion measures $D M$, the observation frequencies $\nu$, the number of added sessions $M$ and the total number $N$ of integrated individual profiles. The pulsar ephemerides are those from the catalogue of Taylor et al. (1995). Pulsars PSR J0030+0451, J0621+1002, J17441134, J1911-1114 and J2051-0827 ephemerides were taken from Somer (2000), Camilo et al. (1996a), Bailes et al. (1997), Lorimer et al. (1997) and Stappers et al. (1996). For pulsars PSR J1022+1001, J1024-0719, J1518+4904, J1911-1114 and J2145-0750 values of $\dot{P}$ were taken from Bailes et al. (1997), Lorimer et al. (1997), Sayer et al. (1997) and Stairs et al. (1999). 
Table 2. Flux density and spectral index

\begin{tabular}{|c|c|c|c|c|c|}
\hline$\overline{\mathrm{PSR}}$ & $\begin{array}{c}F \\
\text { mJy }\end{array}$ & $\begin{array}{l}\Delta F \\
\mathrm{mJy}\end{array}$ & $\alpha$ & $\sigma_{\alpha}$ & References \\
\hline J0030+0451 & 380 & 200 & -2.6 & 0.3 & 1 \\
\hline J0034-0534 & 250 & 120 & -2.3 & 0.1 & $2,4,5$ \\
\hline J0218+4232 & 270 & 150 & -2.1 & 0.4 & 3,6 \\
\hline J0613-0200 & 240 & 100 & -1.8 & 0.1 & $3,4,7,8,9$ \\
\hline J0621+1002 & 50 & 25 & -1.9 & 0.3 & $3,9,10,11$ \\
\hline $\mathrm{J} 0751+1807$ & 70 & 30 & -1.6 & 0.2 & $8,9,11,12$ \\
\hline $\mathrm{J} 1012+5307$ & 30 & 15 & -1.7 & 0.2 & $3,5,8,9,11,13$ \\
\hline $\mathrm{J} 1022+1001$ & 90 & 40 & -1.4 & 0.1 & $3,8,9,10,11,14$ \\
\hline J1024-0719 & 200 & 100 & -1.9 & 0.2 & $4,8,9,11,15$ \\
\hline $\mathrm{B} 1257+12$ & 150 & 50 & -1.8 & 0.2 & $8,5,9,16$ \\
\hline $\mathrm{B} 1310+18$ & 60 & 40 & -2.8 & - & 17 \\
\hline $\mathrm{J} 1518+4904$ & 40 & 20 & -1.3 & 0.1 & $3,8,9,11$ \\
\hline B1534+12 & 110 & 60 & -1.9 & 0.5 & $8,9,11,18$ \\
\hline B1620-26 & 450 & 200 & -2.2 & 0.1 & $3,8,11,19,20$ \\
\hline $\mathrm{J} 1639+36 \mathrm{~A}$ & 70 & 40 & -2.5 & - & 9,17 \\
\hline $\mathrm{B} 1640+2224$ & 450 & 200 & -2.0 & 0.1 & $8,9,11$ \\
\hline $\mathrm{J} 1713+0747$ & 250 & 100 & -1.4 & 0.1 & $3,5,8,9,11,14,21$ \\
\hline J1730-2304 & 310 & 120 & -1.7 & 0.1 & $3,4,7,8$ \\
\hline J1744-1134 & 220 & 100 & -1.8 & 0.1 & $3,4,8,11,15$ \\
\hline B1855+09 & 450 & 250 & -1.6 & 0.1 & $\begin{array}{c}5,8,9,11,19,22 \\
23,24,25\end{array}$ \\
\hline J1911-1114 & 260 & 130 & -2.3 & 0.2 & $3,4,25$ \\
\hline $\mathrm{J} 2019+2425$ & 190 & 100 & -1.9 & - & 9,26 \\
\hline $\mathrm{J} 2033+1736$ & 70 & 30 & -2.6 & - & 9,27 \\
\hline J2051-0827 & 250 & 100 & -1.8 & 0.2 & $3,4,8,11,28$ \\
\hline $\mathrm{B} 2127+11 \mathrm{C}$ & 60 & 40 & -3.3 & - & 9,29 \\
\hline $\mathrm{J} 2145-0750$ & 480 & 120 & -1.6 & 0.1 & $\begin{array}{c}2,3,4,5,8,9,11 \\
14,30\end{array}$ \\
\hline $\mathrm{J} 2229+2643$ & 60 & 30 & -1.8 & 0.4 & $8,9,31$ \\
\hline $\mathrm{J} 2235+1506$ & 170 & 80 & -2.9 & - & 9,31 \\
\hline $\mathrm{J} 2317+1439$ & 90 & 50 & -1.2 & 0.1 & $8,9,31$ \\
\hline $\mathrm{J} 2322+2057$ & 75 & 40 & -2.3 & - & 31 \\
\hline
\end{tabular}

References: 1- Somer (2000), 2 -Bailes et al. (1994), 3 -Stairs et al. (1999), 4 - Toscano et al. (1998), 5- Shitov (1996), 6- Navaro et al. (1995), 7 - Lorimer et al. (1995), 8 -Kramer et al. (1998), 9- Malofeev et al. (2000), 10 -Camilo et al. (1996a), 11- Kramer et al. (1999), 12 -Lundgren et al. (1995), 13 -Nicastro et al. (1995). 14- Kijak et al. (1997), 15 -Bails et al. (1997), 16 -Wolszczan \& Frail (1992), 17- Kulkarni et al. (1991), 18- Wolszczan (1991), 19 -Foster et al. (1991), 20- Lyne et al. (1988), 21- Foster et al. (1993), 22-Kuzmin et al. (1990); 23- Siegelstein (1986), 24 -Fomalont et al. (1992), 25 -Lorimer et al. (1997), 26- Taylor et al. (1995), 27- Ray et al. (1996), 28- Stappers et al. (1996), 29- Anderson et al. (1990), 30- Kuzmin \& Losovsky (1996), 31- Camilo et al. (1996b).

\section{Flux densities}

Measurements of MSP flux densities $F_{\text {PSR }}$ were performed against discrete sources with known flux densities, which were compared by their signal-to-noise ratio $S / N$

$$
\begin{aligned}
F_{\mathrm{PSR}}= & F_{\mathrm{Ref}} \times\left((S / N)_{\mathrm{PSR}} /(S / N)_{\mathrm{Ref}}\right) \\
& \times\left((\Delta f \tau)_{\mathrm{Ref}}^{1 / 2} k_{\mathrm{Ref}}\right) /\left((\Delta f \tau)_{\mathrm{PSR}}^{1 / 2} k_{\mathrm{PSR}} k_{\mathrm{Form}}\right) \\
= & \delta S \times(S / N)_{\mathrm{PSR}} /\left((\Delta f \tau)_{\mathrm{PSR}}^{1 / 2} k_{\mathrm{PSR}} k_{\mathrm{Form}}\right) .
\end{aligned}
$$

Here $F_{\mathrm{PSR}}$ and $F_{\text {Ref }}$ are the flux densities, $(S / N)_{\mathrm{PSR}}$ and $(S / N)_{\text {Ref }}$ the signal-to-noise ratios of the measured pulsar (at the pulse peak) and the reference source, $k_{\text {Ref }}=$ $\sin h_{\text {Ref }}$ and $k_{\mathrm{PSR}}=\sin h_{\mathrm{PSR}}$ are the coefficients that account for the dependence of the antenna effective area for a height $h$ of the reference source and the pulsar respectively. $\Delta f$ is the receiver bandwidth, $\tau$ is the signal integration time, $k_{\mathrm{Form}}$ is the form factor of the pulsar profile for converting the pulse peak flux to the pulsar period-averaged flux and

$\delta S=F_{\text {Ref }}(S / N)_{\text {Ref }}^{-1}(\Delta f \tau)_{\text {Ref }}^{1 / 2} k_{\text {Ref }}$

is a calibration parameter, which characterizes the radiotelescope fluctuation sensitivity (in units of flux density).

Since the noise of the receiving system depends on the Galactic-background brightness temperature $T_{\mathrm{b}}$, we present this calibration parameter as

$\delta S=\delta S_{1000}\left(a+b T_{\mathrm{b}}\right)$.

Here $\delta S_{1000}$ is the radio telescope fluctuation sensitivity toward a sky region with a brightness temperature of $1000 \mathrm{~K}$, taken as the standard. Then, the measured pulsar flux density is

$F_{\mathrm{PSR}}=\delta S_{1000}\left(a+b T_{\mathrm{b}}\right)(S / N)_{\mathrm{PSR}} /\left((\Delta f \tau)_{\mathrm{PSR}}^{1 / 2} k_{\mathrm{PSR}} k_{\mathrm{Form}}\right)$.

Using 70 discrete sources located in sky regions of different Galactic-background temperatures, we have calibrated the radio telescope sensitivity and determined $\delta S_{1000}=115 \pm$ $25 \mathrm{mJy} / \mathrm{MHz} / \mathrm{s}, a=0.4 \pm 0.1$ and $b=0.0006 \pm 0.0001$ for $102 \mathrm{MHz}$ and $\delta S_{1000}=120 \pm 40 \mathrm{mJy} / \mathrm{MHz} / \mathrm{s}$ for $111 \mathrm{MHz}$. The flux densities of the reference sources were obtained from the $86 \mathrm{MHz}$ measurements of Artyukh et al. (1968), converted to our frequencies (102 and $111 \mathrm{MHz}$.)

These measured $\delta S_{1000}, a$ and $b$ are in good agreement with theoretical estimates. For the antenna effective area $A \approx 35000 \sin h \mathrm{~m}^{2}$, antenna-feeder efficiency $\eta \approx 0.7$, antenna stray factor $\beta \approx 0.3$ and the receiver noise factor $N=1.5$ we evaluate $\delta S_{1000} \approx 100 \mathrm{mJy} / \mathrm{MHz} / \mathrm{s}, a \approx 0.4$ and $b \approx 0.0006$.

Measured flux densities $F$ for 30 MSPs are presented in Col. 2 of Table 2. The errors $\Delta F$ (Col. 3 of Table 2) were determined as the square root of the sum of the systematic and random errors squared $\sqrt{ }\left(\sigma_{\text {syst }}^{2}+\sigma_{\text {rend }}^{2}\right)$. We took the systematic errors to be equal to the error in the radio-telescope fluctuation sensitivity $\sigma_{\text {syst }}=25$ and $40 \%$ for 102 and $111 \mathrm{MHz}$ respectively. The random error was estimated as a scatter of the results of different observation sessions. Therefore, it accounts for the scintillation effect as well.

To verify our technique of measurements by the signalto-noise ratio method, we made control measurements by the same method of the flux densities of normal pulsars and compared them to those obtained by a standard method using a noise generator. A comparison of our measurements for 40 normal pulsars with that obtained using the noise generator (Izvekova et al. 1981; Malofeev 1993) shows that they are in a good agreement. 
Recent measurements of the flux density of MSPs by Malofeev et al. (2000) at $111 \mathrm{MHz}$ are also in good agreement with our MSP flux density values as well as Shitov's (1996) MSP data at $103 \mathrm{MHz}$.

\section{Spectra}

Based on these flux density measurements and higher frequency data borrowed from the literature, we have constructed the spectra of MSPs.

Figures 1 and 2 show the spectra of 30 MSPs constructed from our flux density measurements and published data. Our data are represented by open circles.

Spectra of MSPs are very different from those of normal pulsars. In contrast to the expected low-frequency spectral turn-over at sufficiently high frequencies $\left(\nu_{\max }>\right.$ $300 \mathrm{MHz}$ ), our observations do not reveal such an event even at frequencies as low as $102 \mathrm{MHz}$. The low-frequency turn-over appears only in one pulsar, PSR J1012+5307, with a spectrum maximum near $100 \mathrm{MHz}$. At the same time, for the period of this pulsar, $P=5.25 \mathrm{~ms}$, the maximum of the spectrum was expected at $\nu \approx 800 \mathrm{MHz}$, which is not observed. Formally, spectral turn-over can also be noted in the pulsar PSR B1534+12. Further fluxdensity measurements of this pulsar at high frequencies are required. For the remaining pulsars, the spectra are represented by a simple power law $F \propto \nu^{\alpha}$ and exhibit no low-frequency spectral turn-over.

Power-low best fit $F \propto \nu^{\alpha}$ lines are shown for each object, where $\alpha$ is the spectral index. The values of their spectral indices $\alpha$ are given in Col. 4 of Table 2. The errors in the spectral indices $\sigma_{\alpha}$ (Col. 5) are the formal fitting errors. Column 6 of the table lists references to the other flux density measurements which we used to construct the spectra.

For the pulsar PSR J1012+5307, we fitted the spectrum by a power law with a quadratic term, $\log F=$ $c+\alpha \log \nu+\beta \log \nu^{2}$. Table 2 cites the value of the linear term $\alpha$. The quadratic term for this pulsar gives $\beta=-1.0 \pm 0.2$.

The mean spectral index for $23 \mathrm{MSPs}$, for which the flux density was measured at three or more frequencies, $\bar{\alpha}=-1.7 \pm 0.3$, does not differ from $\bar{\alpha}=-1.8 \pm 0.1$ obtained by Kramer et al. (1998) at higher frequencies. There is no significant difference between the mean spectral indices of millisecond and normal pulsars.

Spectra of 7 out of 30 pulsars in our sample are based on only two frequencies. The values of their spectral indices need further refinement by measurements in a wider frequency range. Nevertheless, it is significant that spectra of these pulsars also do not indicate a low frequency turn-over.

\section{Luminosity}

One of the main parameters of pulsar radio emission is the luminosity. However, measurements and comparative studies of luminosity of MSPs have not often been performed. They are obtained only at high frequencies and only the monochromatic luminosity $L_{\nu}=F_{\nu} d^{2}$ was determined, where $F_{\nu}$ - is the flux density at frequency $\nu$ (in $\mathrm{mJy}$ ) and $d$ - the distance to pulsar (in kpc). Kramer et al. (1998) determined the monochromatic luminosity of a set of MSPs at $1.4 \mathrm{GHz}$. They claimed that the monochromatic luminosity of MSPs is about one order of magnitude less than that of normal pulsars.

The monochromatic luminosity is a convenient parameter allowing a comparison of intensities of pulsar radio emission, but does not characterize the full energy of their radio emission. The full energy of radio emission, which one requires to study the mechanism of radio emission, is determined by an integral luminosity over a wide range of radio frequencies $\nu_{1} \div \nu_{2}$

$L_{\text {int }}=\Omega \mathrm{d}^{2} \int_{\nu_{1}}^{\nu_{2}} F_{\nu} \mathrm{d} \nu$.

Here $\Omega$ is the solid angle of the pulsar radiation beam. For an axisymmetric beam, $\Omega=2 \pi\left(1-\cos \left(W_{\text {eff }} / 2\right)\right)$, where $W_{\text {eff }}$ is the effective pulse width.

The spectra of MSPs can be largely described by a single power law $F=F_{0}\left(\nu / \nu_{0}\right)^{\alpha}$. Then, $L_{\text {int }}=\Omega d^{2} F_{0}\left(\nu_{1}^{1+\alpha}-\right.$ $\left.\nu_{2}^{1+\alpha}\right) / \nu_{0}^{\alpha}(1+\alpha)$. For MSPs $1+\alpha<0$, therefore $\nu_{2}^{1+\alpha} \ll$ $\nu_{1}^{1+\alpha}$. We adopted $\nu_{1}=\nu_{0}=100 \mathrm{MHz}$. Then

$L_{\text {int }}=2 \pi\left(1-\cos \left(W_{\text {eff }} / 2\right)\right) d^{2} F_{0} \nu_{0} /(1+\alpha)$.

Since $1 \mathrm{mJy}=10^{-29} \mathrm{~W} / \mathrm{m}^{2} / \mathrm{Hz} / \mathrm{s}=10^{-22} \mathrm{erg} / \mathrm{m}^{2} / \mathrm{Hz} / \mathrm{s}$ and $1 \mathrm{kpc}=3.08510^{19} \mathrm{~m}$, one obtains the integral luminosity in the range of radio frequencies above $\nu=$ $100 \mathrm{MHz}$ :

$L_{\text {int }}=5.9810^{25} d^{2} F_{0}\left(1-\cos \left(W_{\text {eff }} / 2\right)\right) /(1+\alpha)(\mathrm{erg} / \mathrm{s})$.

Based on our measurements of flux density and our spectra, we derived both the monochromatic luminosity at $100 \mathrm{MHz} L_{100}=F_{100} d^{2}$ and the integral luminosity $L_{\text {int }}$ (in $\geq 100 \mathrm{MHz}$ frequency range). Results are listed in Table 3. For PSR J1744-1134, the $d$ value was obtained from parallax measurements by Toscano et al. (1999).

\section{Discussion}

\subsection{Spectra}

A distinctive feature of the spectra of MSPs is the absence of a low-frequency turn-over or, at least, its considerable shift towards low frequency, in comparison with the dependence $\nu_{\max }=120 P^{-0.36}$ observed for normal pulsars (Malofeev \& Malov 1980; Malofeev 1996).

We suggest that the absence of a low-frequency turnover in spectra of MSPs may be attributed to a difference between the magnetic-field structures (topologies) of millisecond and normal pulsars. Kuzmin \& Solov'ev (1986b) showed by model calculation that the pulsar low-frequency spectral turn-over is caused by purely geometric factors, namely, by divergence of magnetic field lines. 
A. D. Kuzmin and B. Ya. Losovsky: Spectra of millisecond pulsars
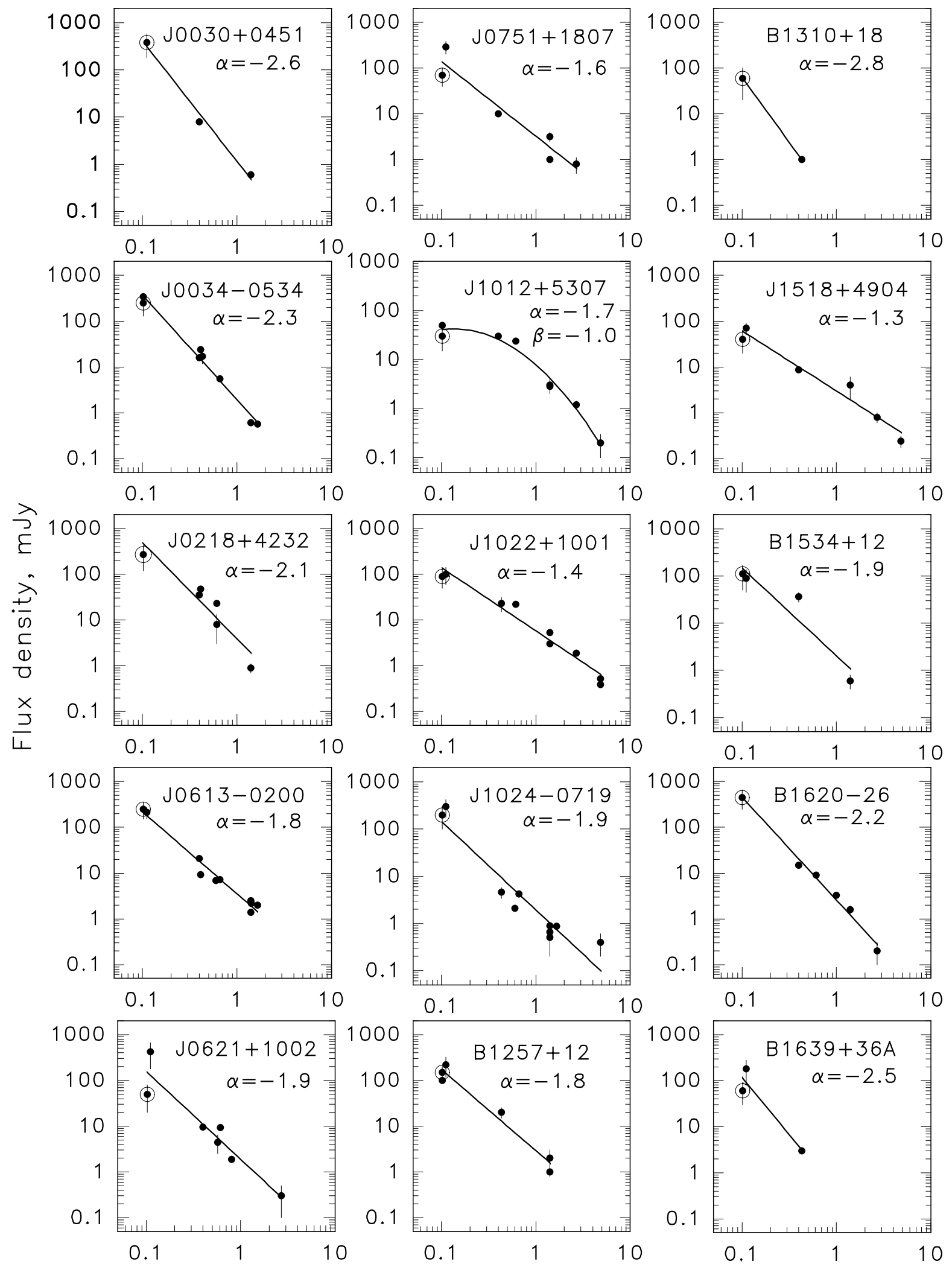

Frequency, GHz

Frequency, GHz

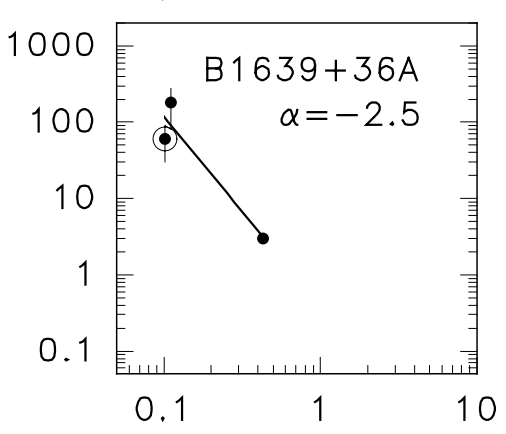

Frequency, GHz

Fig. 1. The spectra of millisecond pulsars PSR J0030+0451 $\div$ B1639+36A. The circled points denote our measurements, the filled points indicate published data. The solid lines represent the regression $F \propto \nu^{\alpha}$. For the pulsar PSR J1012+5307 the spectrum was fitted by a power law with a quadratic term $\log F=c+\alpha \times \log \nu+\beta \log \nu^{2}$ 
A. D. Kuzmin and B. Ya. Losovsky: Spectra of millisecond pulsars
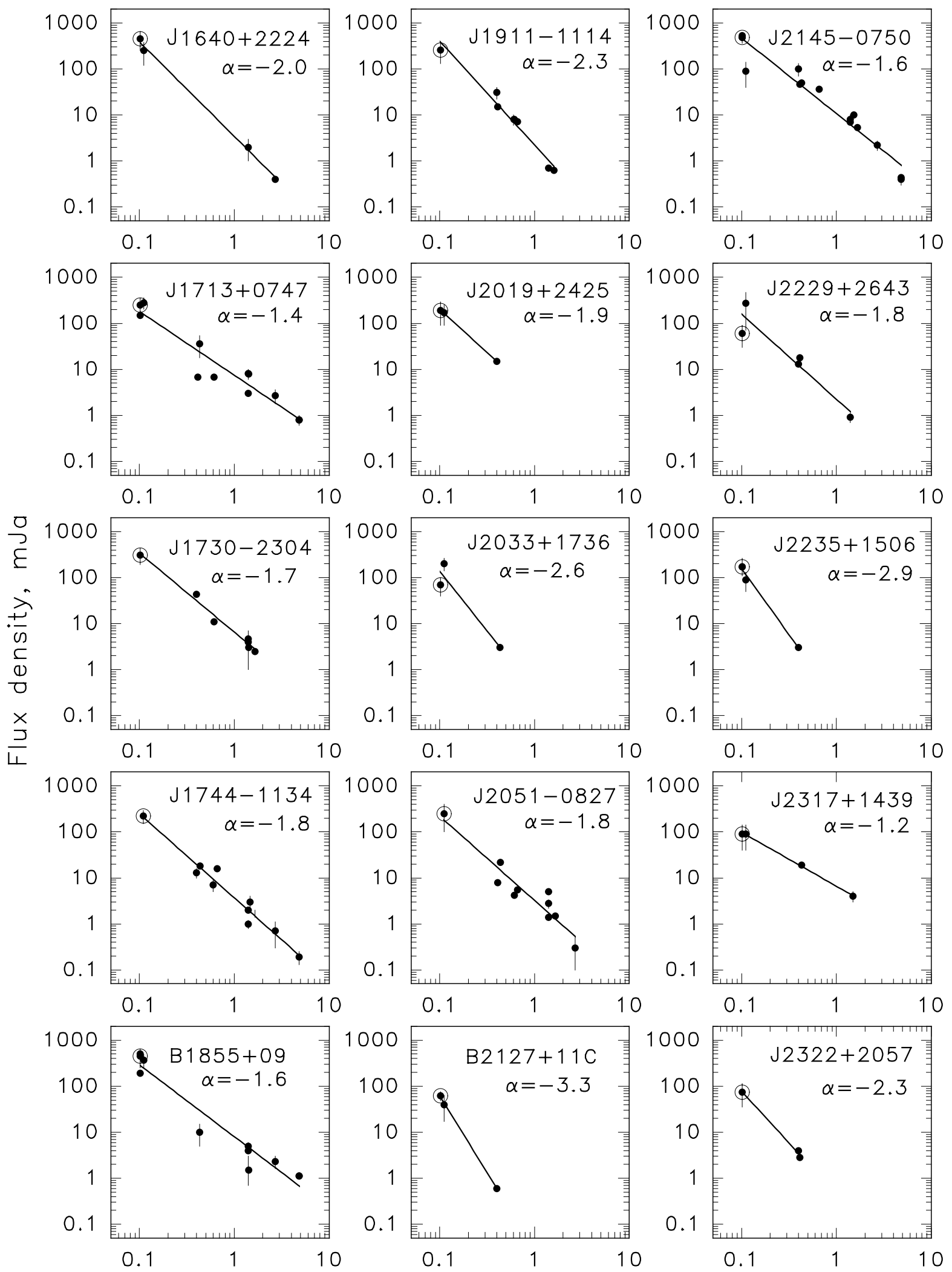

Frequency, GHz

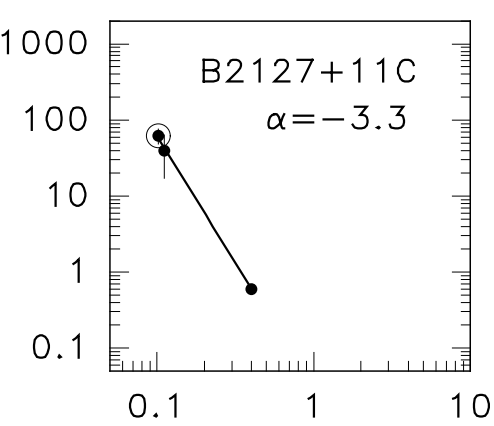

Frequency, GHz

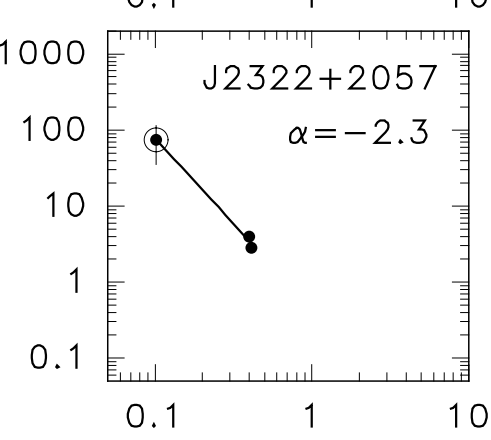

Frequency. GHZ

Fig. 2. The spectra of millisecond pulsars PSR J1640+2224 $\div \mathrm{J} 2232+2057$. The circled points denote our measurements, the filled points indicate published data. The solid lines represent the regression $F \propto \nu^{\alpha}$ 
Table 3. Luminosity

\begin{tabular}{cccc}
\hline PSR & $\begin{array}{c}d \\
\mathrm{kpc}\end{array}$ & $\begin{array}{c}\log L_{100} \\
\mathrm{mJy} \mathrm{kpc}^{2}\end{array}$ & $\begin{array}{c}\log L_{\text {int }} \\
\mathrm{erg} / \mathrm{s}\end{array}$ \\
\hline $\mathrm{J} 0030+0451$ & 0.23 & 1.3 & 27.1 \\
$\mathrm{~J} 0034-0534$ & 0.98 & 2.4 & 28.3 \\
$\mathrm{~J} 0218+4232$ & $\geq 5.85$ & $\geq 3.9$ & $\geq 30.0$ \\
$\mathrm{~J} 0613-0200$ & 2.19 & 3.1 & 29.2 \\
$\mathrm{~J} 0621+1002$ & 1.67 & 2.1 & 27.2 \\
$\mathrm{~J} 0751+1807$ & 2.02 & 2.5 & 28.6 \\
$\mathrm{~J} 1012+5307$ & 0.52 & 0.9 & 27.0 \\
$\mathrm{~J} 1022+1001$ & 0.60 & 1.5 & 27.6 \\
$\mathrm{~J} 1024-0719$ & 0.35 & 1.4 & 27.5 \\
$\mathrm{~B} 1257+12$ & 0.62 & 1.8 & 27.7 \\
$\mathrm{~B} 1310+18$ & 18.9 & 4.3 & 29.7 \\
& $\geq 1.8$ & $\geq 2.3$ & $\geq 27.6$ \\
$\mathrm{~J} 1518+4904$ & 0.70 & 1.3 & 27.1 \\
$\mathrm{~B} 1534+12$ & 0.68 & 1.7 & 27.2 \\
$\mathrm{~B} 1620-26$ & 1.8 & 3.1 & 28.8 \\
$\mathrm{~J} 1639+36 \mathrm{~A}$ & 7.7 & 3.6 & 29.3 \\
& $\geq 2.7$ & $\geq 2.7$ & $\geq 28.4$ \\
$\mathrm{~B} 1640+2224$ & 1.18 & 2.8 & 28.9 \\
$\mathrm{~J} 1713+0747$ & 1.10 & 2.5 & 28.9 \\
$\mathrm{~J} 1730-2304$ & 0.51 & 1.9 & 28.1 \\
$\mathrm{~J} 1744-1134$ & 0.36 & 1.5 & 27.2 \\
$\mathrm{~B} 1855+09$ & 0.90 & 2.6 & 28.7 \\
$\mathrm{~J} 1911-1114$ & 1.6 & 2.8 & 28.4 \\
$\mathrm{~J} 2019+2425$ & 0.91 & 2.2 & 28.4 \\
$\mathrm{~J} 2033+1736$ & 1.38 & 2.1 & 27.8 \\
$\mathrm{~J} 2051-0827$ & 1.28 & 2.6 & 28.6 \\
$\mathrm{~B} 2127+11 \mathrm{C}$ & 10.0 & 3.8 & 28.9 \\
$\mathrm{~J} 2145-0750$ & 0.50 & 2.1 & 27.8 \\
$\mathrm{~J} 2229+2643$ & 1.43 & 2.1 & 28.1 \\
$\mathrm{~J} 2235+1506$ & 1.15 & 2.4 & 27.7 \\
$\mathrm{~J} 2317+1439$ & 1.9 & 2.5 & 29.1 \\
$\mathrm{~J} 2322+2057$ & 0.78 & 1.7 & 27.4 \\
\hline & & & \\
& &
\end{tabular}

This geometrical interpretation of the nature of the turn-over can explain also the absence of this feature in MSPs. The radius of the light cylinder of MSPs is much smaller than for normal pulsars. This means that an emission region is located closer to the stellar surface than in normal pulsars. This may modify the magnetic field dominating the radio emission region, altering its configuration from a pure dipole to a higher order multipole (Davies et al. 1984; Kuzmin et al. 1986b). Multipole components near the stellar surface have been assumed by Ruderman \& Sutherland (1975), Ruderman (1991), Chen \& Ruderman (1993) and Krolic (1999). In a multipole field, configurations with a small divergence of the cone of open field lines are possible (see for example Fig. 5 in Kuzmin \& Losovsky 1996). This small divergence of the magnetic field lines may inhibit low-frequency spectral turn-over.

This interpretation is consistent with the weak frequency dependence of the integrated-profile width for MSPs, which also suggests a difference in the magneticfield structures (topologies) of millisecond and normal pulsars (Kuzmin \& Losovsky 1999a, 1999b).

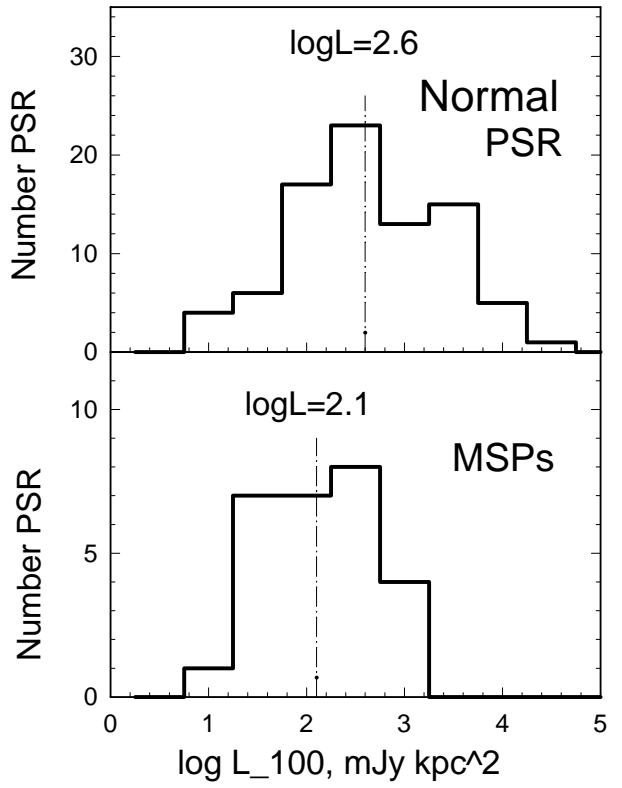

Fig. 3. The luminosity distribution of normal and millisecond pulsars. The mean values of $\log \bar{L}=2.6$ for normal and $\log \bar{L}=$ 2.1 for millisecond pulsars are shown

An alternative or complementary interpretation is that the emission region is very compact (Kramer et al. 1998). In this case, the divergence of field lines is also small and one may expect that there is no low-frequency turn-over.

Another possibility is the conception of two types of pulsars (Malov 1989), according to which the emission of short-period pulsars is generated near the light cylinder and emerges from approximately the same level irrespective of frequency. In this case, the low-frequency turn-over can also be less pronounced or absent altogether.

\subsection{Luminosity}

We note that the luminosities of PSR B1310+18 and PSR B1639+36A, $\log L_{100}=4.3$ and 3.6, which are based on the Taylor et al. (1995) catalog values of distance $d=18.9$ and $7.7 \mathrm{kpc}$ respectively, are more than one order of magnitude higher that the mean value $\log \bar{L}_{100}=2.3$. These distances are based on the suggestion by Kulkarni et al. (1991) that PSR B1310+18 and PSR B1639+36A are located in globular clusters M 53 (NGC 5024) and M 13 (NGC 6205), respectively. But this identification is based only on a coincidence of their coordinate positions and may not be reliable. Estimation of their distances from their dispersion measures, using the Taylor \& Cordes (1993) model, yield $d \geq 1.8$ and $\geq 2.7 \mathrm{kpc}$, corresponding to luminosities $\log L_{100} \geq 2.3$ and $\geq 2.7$ close to the mean value $\log \bar{L}_{100}=2.3$. In Table 3 we list PSR B1310+18 and PSR B1639+36A for both these values. The distance and luminosity of these pulsars in globular clusters should probably be treated as questionable until confirmed.

We suggest also that one needs to refine the Taylor et al. (1995) catalog values of distancesd $\geq 5.85 \mathrm{kpc}$ 

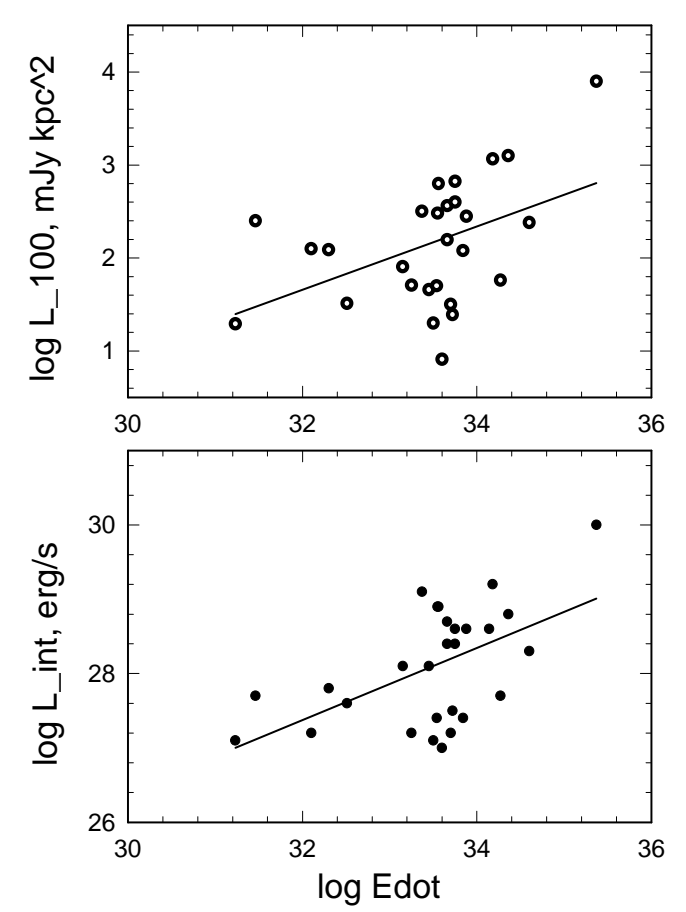

Fig. 4. Monochromatic (upper picture) and integral (lower picture) luminosity dependence versus the losses of rotation energy $\dot{E}$. Solid lines represent the regression $L \propto \dot{E}^{\beta}$

and $d=10.0 \mathrm{kpc}$, that lead to high values of luminosity, $\log L_{100} \geq 3.9$ and $\log L_{100}=3.8$, for the MSPs PSR J0218+4232 and PSR B2127+11C, respectively.

The luminosity distribution of normal and millisecond pulsars (omitting the above-mentioned PSR J0218+4232, $\mathrm{B} 1310+18, \mathrm{~B} 1639+36 \mathrm{~A}$ and $\mathrm{B} 2127+11 \mathrm{C}$, whose distances and luminosities are questionable) is shown in Fig. 3. The mean value of MSPs luminosity $\log \bar{L}_{100 \mathrm{MSP}}=2.1 \pm 0.6$ is a little less than that for our sample of normal pulsars $\log \bar{L}_{100 \text { norm }}=2.6 \pm 0.8$.

On the other hand, it is interesting to note that if the Taylor et al. (1995) catalog values of distances $d$ are correct, then MSPs, which are detected in globular cluster, are of unusually high luminosity. For all 4 MSPs studied here the mean value of luminosity is $\log \bar{L}_{100}=3.7 \pm 0.5$, compared to the above-mentioned $\log \bar{L}_{100 \mathrm{MSP}}=2.1 \pm 0.6$ for other MSPs.

We have analyzed the dependence of the MSPs luminosity on other parameters. Luminosity depends on the rate of loss of rotation energy $\dot{E}$ as

$\log L_{100}(\dot{E})=-10.0( \pm 4.5)+0.36( \pm 0.13) \log \dot{E}$

for monochromatic and

$\log L_{\text {int }}(\dot{E})=11.8( \pm 4.8)+0.48( \pm 0.14) \log \dot{E}$

for integral luminosity. These relationships are shown in Fig. 4.

The conversion efficiency of rotation energy to radio luminosity $\eta=L_{\text {int }} / \dot{E}$ is found to be about $10^{-5}$, decreasing with $\dot{E}$ as $\eta \propto \dot{E}^{-0.5 \pm 0.2}$.

No other significant dependencies of luminosity on other pulsar parameters were found.

\section{Summary}

We have measured the flux densities of 30 millisecond pulsars at the lowest frequency of 102 and $111 \mathrm{MHz}$. Using published data, we have constructed their spectra in the broadest frequency range from $102 \mathrm{MHz}$ to $4.8 \mathrm{GHz}$.

The spectra of millisecond pulsars do not exhibit the low-frequency spectral turn-over which is typical of most normal pulsars.

We explain the absence of low-frequency spectral turnover in millisecond pulsars by a difference in the magneticfield structures (topologies) of millisecond and normal pulsars.

There is no significant difference between the mean spectral indices of millisecond and normal pulsars.

The luminosity of millisecond pulsars is less than that of normal ones. Luminosity decreases with increased rate of loss of rotation energy.

Acknowledgements. The authors thank the staff of the BSA radio telescope and especially V. V. Ivanova, A. S. Alexandrov, K. A. Lapaev and V. D. Pugachev for providing observations and data reduction. We are grateful to F. G. Smith and the anonymous referee for valuable comments and perfection of the English. The authors thank also V. M. Malofeev for providing his data of flux density measurements of millisecond pulsars and A. Somer for the PSR J0030+0451 ephemeres before publication. This study was supported in part by the Russian Foundation for Basic Research (project No. 98-02-17532) and INTAS (project No. 96-0154).

\section{References}

Anderson, S. B., Gorham, P. W., Kulkarni, S. R., et al. 1990, Nature, 346, 42

Artyukh, V. S., Vitkevitch, V. V., Dagkesamansky, R. D., et al. 1968, SvA, 45, 712

Bailes, M., Harison, P. A., Lorimer, D., et al. 1994, ApJ, 425, L41

Bailes, M., Johnston, S., Bell, J. F., et al. 1997, ApJ, 481, 386

Bruk, Yu. M., Davies, J. G., Kuzmin, A. D., et al. 1978, AZh, 55,1031

Camilo, F., Nice, D. J., Shrauner, J. A., et al. 1996a, ApJ, 461, 812

Camilo, F., Nice, D. J., \& Taylor, J. H. 1996b, ApJ, 469, 819

Chen, K., \& Ruderman, M. A. 1993, ApJ, 408, 179

Davies, J. G., Lyne, A. G., Smith, F. G., et al. 1984, MNRAS, 211,57

Erickson, W .C., \& Mahoney, M. J. 1985, ApJ, 299, L29

Fomalont, E. B., Goss, W. M., \& Lyne, A. G. 1992, MNRAS, 258, 497

Foster, R. S., Fairhead, L., \& Backer, D. C. 1991, ApJ, 378, 687

Foster, R. S., Wolszczan, A., \& Camilo, F. 1993, ApJ, 410, L91

Foster, R. S., Cadwell, B. J., Wolszczan, A., et al. 1995, ApJ, 454,826

Gupta, Y., Rickett, B. J., \& Coles, W. A. 1993, ApJ, 402, 183

Izvekova, V. A, Kuzmin, A. D, Malofeev, V. M., et al. 1979, Aust. J. Phys., 32, 25

Izvekova, V. A., Kuzmin, A. D., Malofeev, V. M., et al. 1981, Ap\&SS, 78, 45 
Kijak, J., Kramer, M., Wielebinski, R., et al. 1997, ApJ, 318, L63

Kramer, M., Xilouris, K. M., Lorimer, D. R., et al. 1998, ApJ, 501,270

Kramer, M., Lange, C., Lorimer, D., et al. 1999, ApJ, 526, 975

Krolic, J. H. 1999, ApJ, 373, L69

Kulkarni, S. R., Anderson, S. B., Prince, T. A., et al. 1991, Nature, 349, 47

Kuzmin, A. D., Malofeev, V. M., Shitov, Yu. P., et al. 1978, MNRAS, 185, 441

Kuzmin, A. D., \& Soloviev, A. G. 1986, SvA, 30, 38

Kuzmin, A. D., Alexeev, Yu. I., Lapaev, K. A., et al. 1990, Astron. Lett., 16, 208

Kuzmin, A. D., \& Losovsky, B. Ya. 1996, A\&A, 308, 91

Kuzmin, A. D., \& Losovsky, B. Ya. 1999, Astron. Rep., 43, 288

Kuzmin, A. D., \& Losovsky, B. Ya. 1999, Astron. Lett., 25, 441

Kuzmin, A. D., \& Losovsky, B. Ya. 2000a, PASPC, 202, 235

Kuzmin, A. D., \& Losovsky, B. Ya. 2000b, Astron. Lett., 26, 500

Lorimer, D. R., Nicastro, L., Lyne, A. G., et al. 1995, ApJ, 430, 933

Lorimer, D. R., Lyne, A. G., Bailes, M., et al. 1997, MNRAS, 283, 1383

Lundgren, S. C., Zepka, A. F., \& Cordes, J. M. 1995, ApJ, 453, 419

Lyne, A. G., Biggs, J. D., Ashwort, M., et al. 1988, Nature, 332,45

Malov, I. F. 1989, in Proc. of Lebedev Phys. Inst., 199, ed. A. D. Kuzmin, Pulsars, (Nova Science Publisher), 111

Malofeev, V. M., \& Malov, I. F. 1980, AZh, 57, 90

Malofeev, V. M. 1993, Astron. Lett., 19, 138

Malofeev, V. M. 1996, PASPC, 105, 271

Malofeev, V. M. 2000, PASPC, 202, 221
Malofeev, V. M., Malov, O. I., \& Shchegoleva, N. V. 2000, Astron. Rep., 44, 436

Navaro, J., deBruyn, A. G., Frail, D. A., et al. 1995, ApJ, 455, L55

Nicastro, L., Lyne, A. G., Lorimer, D. R., et al. 1995, MNRAS, 273, L68

Ray, P. S., Thorset, S. E., Jenet, F. A., et al. 1996, ApJ, 470, 1103

Ruderman, M. A., \& Sutherland, P. G. 1975, ApJ, 196, 51

Ruderman, M. A. 1991, ApJ, 382, 576

Sayer, R. W., Nice, D. J., \& Taylor, J. H. 1997, ApJ, 474, 426

Siegelstein, D. J. 1986, Nature, 322, 714

Shitov, Yu. P. 1996, Unpublished work

Sieber, W. 1973, A\&A, 28, 273

Somer, A. 2000, PASPC, 202, 17

Stairs, I. H., Thorset, S. E., Camilo, F., et al. 1999, ApJS, 123 672

Stappers, I. H., Bailes, M., Lyne, A. G., et al. 1996, ApJ, 465, L119

Stinebring, D. R., Smirnova, T. V., Hankins, T. H., et al. 2000, ApJ, 539, 300

Taylor, J. H., \& Cordes, J. M. 1993, ApJ, 411, 674

Taylor, J. H., Manchester, R. N., Lyne, A. G., et al. 1995, Catalog of 706 pulsars, Unpublished work

Toscano, M., Bailes, M., Manchester, R. N., et al. 1998, ApJ, 506,863

Toscano, M., Britton, M. C., Manchester, R. N., et al. 1999, ApJ, 523, L171

Xilouris, K. M., Kramer, M., Jessner, A., et al. 1998, ApJ, 501, 286

Wolszczan, A. 1991, Nature, 350, 368

Wolszczan, A., \& Frail, D. A. 1992, Nature, 355, 145 